\title{
Synthesis of autonomous robots through evolution
}

\author{
Stefano Nolfi and Dario Floreano
}

\begin{abstract}
Evolutionary robotics is the attempt to develop robots through a selforganized process based on artificial evolution. This approach stresses the importance of the study of systems that have a body and that are situated in a physical environment, and which autonomously develop their own skills in close interaction with the environment. In this review we briefly illustrate the method and the main concept of evolutionary robotics, and examine the most significant contribution in this area. We also discuss some of the contributions that this research area is making to the foundational debate in cognitive science.
\end{abstract}

The basic idea behind evolutionary robotics (the attempt to synthesize robots through evolutionary techniques) sees an initial population of different artificial chromosomes, each encoding the control system (and sometimes the morphology) of a robot, randomly created and put in the environment [1-5]. Each robot is then let free to act (move, look around, manipulate) according to a genetically specified controller and its performance on various tasks is automatically evaluated. The fittest robots are allowed to reproduce by generating copies of their genotypes with the addition of changes introduced by some genetic operators (such as mutations, crossover and duplication). This process is repeated for several generations until an individual is born that satisfies the performance criterion (fitness function) set by the experimenter. Robots might also change during lifetime and therefore might adapt to their environment both phylogenetically and ontogenetically.

This approach stresses the importance of studying systems that, just like natural organisms, have a body and are situated in a physical environment [6-10], and which autonomously develop their own skills in close interaction with the environment. Systems of this sort are difficult to design from the perspective of an external observer. However, they can be obtained either by carefully mimicking natural organisms (as long as a detailed description of their organization is available, see for example Ref. [11]), or by resorting to an automatic process such as artificial evolution that allows them to self-organize autonomously.

In this review we will briefly illustrate three research topics in which experimental studies conducted with this approach are producing significant contributions; namely the synthesis and study of robots that (1) exploit the opportunities that arise from the possibility to interact with an external environment; (2) consist of a control system and a body morphology that are tightly coupled; and
(3) display an ability to adapt to varying environmental conditions. Finally, we will briefly discuss some of the contributions that this research area is making to the foundational debate in cognitive science.

\section{Exploiting the interaction with the environment} The behaviour of embodied and situated organisms is an emergent result of the dynamical interaction between the nervous system, the body and the external environment [12,13]. This simple consideration has several important consequences that are far from being fully understood. One important aspect, for instance, is the fact that motor actions partially determine the sensory pattern that organisms receive from the environment. By coordinating sensory and motor processes organisms can select favourable sensory patterns and thus enhance their ability to achieve their adaptive goals.

Examples of processes falling within this category have been identified in natural organisms. Dill et al. demonstrated that because the fruit fly Drosophila cannot always recognize a pattern appearing at different locations in the retina, the insect solves this problem of shift invariance by moving so to bring the pattern to the same retinal location where it has been presented during the storage process [14]. Franceschini demonstrated that flies use motion to visually identify the depth of perceived obstacles [11]. Moreover, there is evidence that environmental feedback obtained through motor actions plays a crucial role in normal development $[15,16]$.

Instead, in an artificial system, aside from a few notable exceptions [17-19], the potential to design systems that exploit sensory-motor coordination remains largely unexplored. This can be explained by considering that, as stated above, behaviour is the emergent result of the interactions between the individual and the environment. Given that in dynamical systems there is a complex and indirect relationship between the rules that determine the interactions and the emergent result of those interactions, it is very difficult to identify how the interactions between the organism and the external environments contribute to the resulting behaviour (for an introduction to dynamical systems and dynamical approaches to the study of behavior see Ref. [13]). As a result, designing systems that exploit sensory-motor coordination is rather difficult (Ref. [10] details an attempt to identify new design principles that might help to achieve this goal). 
Fig. 1. The miniature mobile Khepera robot has a diameter of $6 \mathrm{~cm}$. It is equipped with two wheels and eight active infrared sensors (six on the front and two on the back) that can measure the distance from objects or the amount of ambient light.

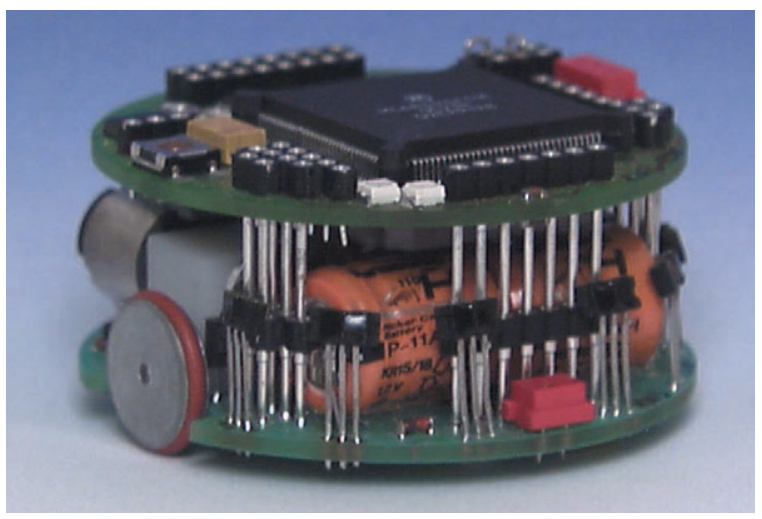

From this point of view evolutionary experiments in which robots autonomously develop their skills in close interaction with the environment represent an ideal framework for studying sensory-motor coordination. Indeed, in most of the experiments conducted with artificial evolution one can observe the emergence of behaviour exploiting sensory-motor coordination to solve difficult tasks. The analysis of evolved robots and the identification of how they exploit the interaction with the environment is often very difficult and requires significant effort, but is generally much simpler than the analysis of natural organisms because the former are much more simple and can be manipulated much more freely than the latter. In addition, such analysis may allow the identification of new explanatory hypotheses that may produce new models of natural behaviour.

By carefully analysing the result of a simple evolutionary experiment, Nolfi showed that evolved individuals exploit behavioural attractors resulting from the interaction between the robot and the environment in order to discriminate between different objects [20,21]. In this experiment the miniature mobile Khepera robot was asked to locate and remain close to a target object [22]. The robot was equipped with six infrared proximity sensors in the front side and two wheels that could rotate in both directions at different speeds (Fig. 1). The environment consisted of an arena of $60 \times 35 \mathrm{~cm}$ surrounded by walls, and contained a cylindrical object with a diameter of $2.3 \mathrm{~cm}$ located at a random position.

The robot's control system was a neural network with six sensory neurons encoding the activation of the six frontal infrared sensors and two output neurons encoding the speeds of the two wheels (for a detailed description of the experiment parameters see Refs [20,21]). By evolving the weights of the neural controllers, the author observed that, after a few generations, evolved individuals were able to find and stay close to the cylindrical object. This implied that evolved robots were capable of discriminating between walls and cylinders.

Further analysis of the problem and of the evolved solutions revealed that the problem of discriminating between sensory patterns belonging to walls and

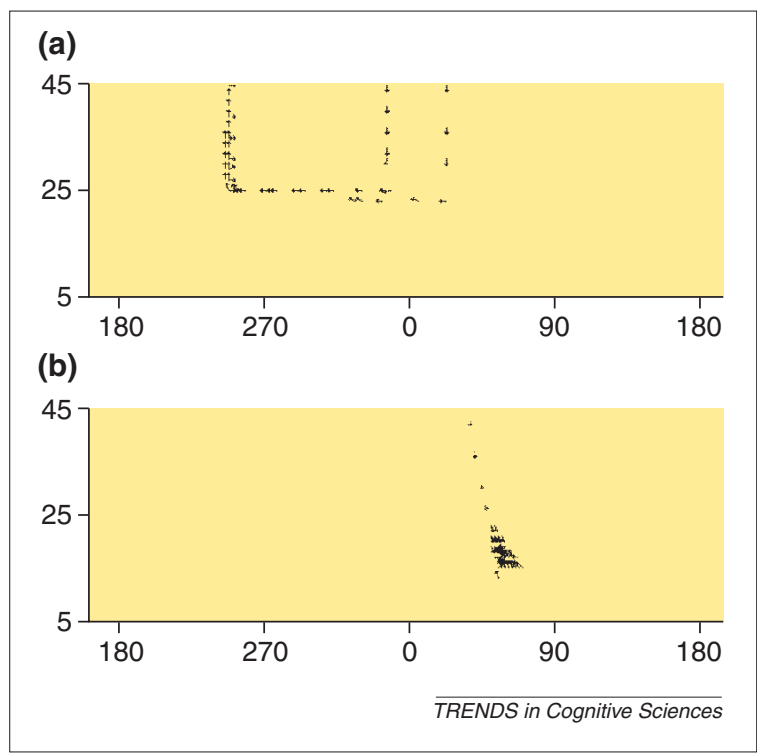

Fig. 2. Angular trajectories of a typical evolved individual close to a wall (a) and to a cylinder (b). The picture was obtained by placing the individual at a random position in the environment, leaving it free to move for 500 sensory-motor cycles, and recording displacements at positions relative to the two types of objects for distances smaller than $45 \mathrm{~mm}$. For sake of clarity, arrows are used to indicate the relative direction but not the amplitude of movements. When the individual reaches a distance of approximately $20 \mathrm{~mm}$ from an object, it avoids walls but approaches cylinders until it reaches the attractor area located at a distance of approximately $15 \mathrm{~mm}$ and an angle of approximately $60 \mathrm{deg}$. The resulting trajectories converge to the centre of the area, allowing the individual to keep more or less the same position relative to the cylinder.

cylinders is far from trivial, given that the sensory patterns corresponding to the two different objects were very similar, and that the discrimination behaviour resulted from a large number of sensory-motor interactions with the environment [20,21]. In particular, evolved individuals acted so to produce behavioural attractors (back and forth and left and right movements that, overall, keep the robot in the same relative position) close to cylinders, but not close to walls (Fig. 2). This experiment showed that artificial evolution can synthesize solutions that are much simpler than those that can be obtained through explicit design [20,21].

Further experiments revealed several other ways in which evolved robots can exploit sensory-motor coordination, so as to:

- increase the frequency of sensory states to which they can react more effectively and reduce the frequency of sensory states to which they react less effectively [23];

- select sensory states in which groups of sensory patterns requiring different motor answers do not strongly overlap [24] (Box 1);

- increase the perceived differences between different objects [25,26];

- select useful learning experiences [25].

\section{Co-evolution of body and brain}

The fact that behaviour results from the interaction between the control system, the robot body, and the 
Box 1. Exploiting sensory-motor coordination to turn hard problems into simpler ones

In a recent paper Clark and Thornton introduced the term Type- 2 problems to denote hard tasks in which the problem of mapping input patterns into suitable output patterns is complicated by the fact that the regularities that can allow such a mapping are marginal or hidden in the sensory patterns (regularities are features of the input patterns that can be used to discriminate between classes of patterns requiring different answers) [a]. On the basis of this consideration, they distinguished Type-2 problems (hard problems) from Type-1 problems (easy problems) in which regularities are directly available in the sensory patterns.

Type-2 problems, which require complex input-output mappings, may be reduced to Type-1 (tractable) problems by recoding input patterns so as to enhance useful regularities [a]. This can be achieved in two ways. One possibility is to recode input patterns internally. Elman, for example, showed how complex tasks that cannot be solved by training a feedforward neural network with back-propagation for the entire task can be solved if the network is first trained on a simpler sub-task and then on the entire task [b]. The first learning phase affects how the sensory patterns are recoded at the level of the internal neural layer [b]. This recoding enhances the regularities of the sensory patterns, and thus reduces the process of learning the entire task from a Type-2 to a Type-1 problem [a].

Another possibility is to exploit sensory-motor coordination. Embodied and situated robots can transform Type- 2 problems into Type-1 problems by actively structuring their own input. Scheier et al. evolved a Khepera robot to approach large (and avoid small) cylindrical objects located in an arena surrounded by walls [24]. Analysis of evolved robots showed that selected behaviours allowed the robot to experience sensory patterns that were easy to discriminate (such that the two groups of sensory patterns corresponding to the two objects were well separated in the input space). To understand this point, we should consider that individuals that act differently may experience different sensory states, and therefore may face simpler or harder discrimination problems. The analysis of different individuals showed that although individuals of the first generation were facing a Type- 2 discrimination problem, after approximately 40 generations individuals were facing a simple Type-1 discrimination problem, in which the two groups of experienced sensory states were nicely separated in the input space. In other words, evolved robots can exploit sensory-motor coordination to simplify the task of discriminating between objects.

\section{References}

a Clark, A. and Thornton, C. (1997) Trading spaces: computation, representation, and the limits of uniformed learning. Behav. Brain Sci. 20, 57-90

b Elman, J.L. (1993) Learning and development in neural networks: the importance of starting small. Cognition 48, 71-99 two systems in natural organisms is probably one of the key reasons for the extraordinary achievements of natural evolution. The tight interaction between the control system and the body morphology complicates the study of adaptive behaviour in natural organisms because it is not always possible to assign credit to individual aspects of observed behaviours as they are effective only when coupled with a given morphology [16].

Some examples of effective coupling between body and brains have been identified in natural organisms. In crickets, for example, the morphology of the body filters and amplifies specific auditory inputs. This allows crickets to easily identify a specific calling song [27-28]. Extra-tympanic structures also play a crucial role in the hearing of vertebrates [16]. The morphology of the body also plays important functional roles on the effect of neural outputs. For instance, 'Muscle acts as a low-pass filter of motor neural outputs; that is, it filters out the high frequency components of the neural outputs. In addition, the mechanical advantage of a muscle and the response of the whole body to the contraction of any particular muscle are a complex function of the geometric relationships and positions of other muscles and joints, and the prior history of activation of that muscle' [16].

Evolutionary robotics experiments in which both the control system and the morphology of the robot are encoded in the genotype and co-evolved can shed light on the complex interplay between body and brains in natural organisms, and may lead to the development of new and more effective artifacts. Although rather few and preliminary experiments have been conducted in this area up to now, this topic is receiving increased attention [29]. Cliff et al. and Harvey et al. co-evolved the control system and the sensory morphology of a mobile robot $[1,30]$. The genotype consisted of two parts encoding the control system (the connection weights and the architecture of a neural controller) and the visual morphology (number, size and position of visual receptive fields), respectively. The authors reported that co-evolution of the two systems allowed evolved robots to rely on very simple strategies, such as comparing the correlated activities of only two visual receptors located at strategic positions on the retinal surface.

Lichtensteiger and Eggenberger evolved the morphology of a compound eye of a robot asked to move on a straight trajectory, and observed that evolved individuals displayed higher density of photoreceptors in the front [31].

Lund et al. co-evolved in simulation the control system and some characteristics of the body of Khepera-like robots that were selected for their ability to navigate while avoiding obstacles [32]. The morphological features that were allowed to change were the diameter of the body, the distance between the two wheels, and the wheel radius. The authors analysed the distribution of evolved robots in the 
Fig. 3. An evolved morphology. The control system consists of a neural network driving the central actuator in perfect anti-phase with the two synchronized lateral limbs, so that while the upper two limbs push the central body is retracted, and vice-versa. Figure courtesy of H. Lipson and J. Pollack.

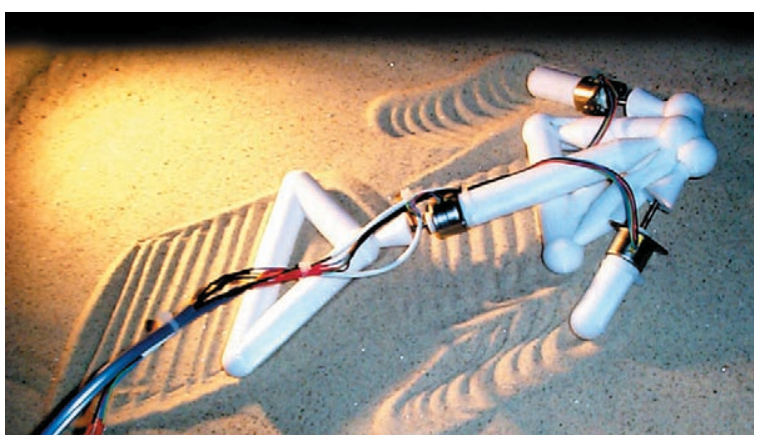

morphological space and observed interesting correlations between evolved morphological characteristics (such as an almost linear relationship between body size and wheel distance), and between morphology and environmental properties (robots evolved in environments with several obstacles were smaller).

Pollack and co-workers have explored the possibility of evolving the morphology of physical robots. In preliminary work, they evolved the shape of functional objects such as bridges and moving cranes in simulation, and later built the evolved structures out of Lego ${ }^{\mathrm{TM}}$ bricks [33]. More recently, they evolved the control system (sigmoid neurons) and morphology of robots composed of linear actuators, bars, and joints [34]. Some evolved individuals were automatically built out of thermoplastic material with a 3-D printer. The printer takes as input the genetic description of the robot morphology and its temperature-controlled head lays down thermoplastic material layer by layer with holes for motors and joints, which are snapped in later. The authors demonstrated this technology in the case of robots evolved for straight navigation in which the fitness function was the distance covered by the robot (Fig. 3). These robots displayed various forms of locomotion, including crawling and forms of walking, both in simulation and in reality.

\section{Evolution of plastic individuals}

In the experiments described, evolving individuals change phylogenetically but not ontogenetically (i.e. they do not change during lifetime). Over the past few years, there has been increasing interest in evolving plastic individuals (individuals that are also capable of adapting during lifetime). These studies have been conducted for two different purposes: (1) to look at the advantages, in terms of performance, obtained by combining these two adaptive techniques; and (2) to understand the role of the interaction between two adaptive processes that resort to different mechanisms and occur at different timescales. The general picture emerging from this body of research is that lifetime adaptation has several functions within an evolutionary perspective [35]:

- It complements evolution by allowing individuals to adapt to environmental changes that take place during the lifetime of the individual or within few

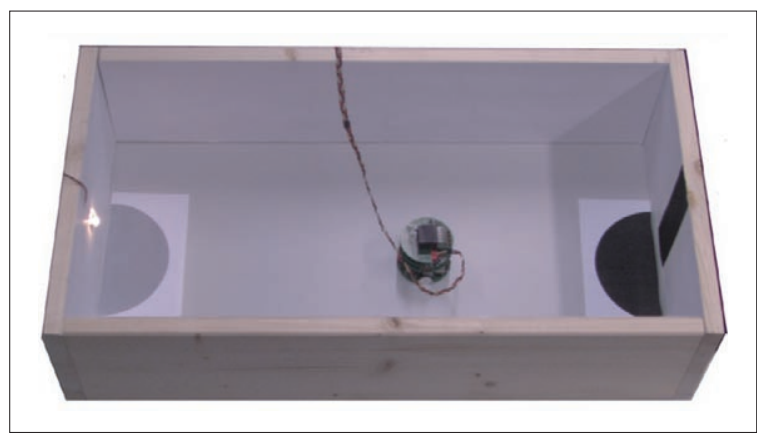

Fig. 4. A mobile Khepera robot equipped with a vision module can gain fitness points by staying on the grey area under the light bulb only when the light is on. The light is normally off, but it can be switched on if the robot passes over the black area positioned on the other side of the arena. The robot can detect ambient light and wall colour, but not the colour of the floor.

generations, and therefore cannot be tracked by evolution $[25,36,37]$. In addition, plastic individuals can adapt to sensory, motor and environmental change that takes place after the evolutionary process [38].

- It can help and guide evolution by channelling the evolutionary process towards promising directions, and it can significantly speed up the synthesis of viable individuals [29-42].

- It might produce more effective behaviours and facilitate the ability to scale-up to problems that involve a larger search space [38].

More generally, this research shows that the interaction between evolution and lifetime adaptation deeply alters both processes. Evolution in interaction with learning displays dynamics that differ significantly from those observed when evolution operates alone. Although in non-plastic individuals the inherited characters are directly selected for their ability to produce successful behaviours, in the case of individuals that learn, the inherited characters are selected for their ability to incorporate a predisposition to learn. Similarly, learning within an evolutionary perspective has rather different characteristics from learning studied in isolation, as in 'traditional' connectionist research.

To better illustrate this point, consider the experiment performed by Urzelai and Floreano in which a Khepera robot provided with a linear array of visual receptors has to perform a sequential task that consists of switching a light on and then moving towards the light bulb [38] (Fig. 4). The robot was controlled by a fully recurrent neural network composed of 12 neurons. Ten sensory neurons received input from four proximity sensors, three ambient light sensors and three areas of the camera, respectively; two motor neurons controlled the speeds of the two wheels. The genotype of the evolving individuals consisted of 12 blocks, each encoding the properties of all the incoming synapses of the corresponding neuron. These properties consisted in the sign of the synapses, the adaptation rule (one of four possible Hebb learning rules) and the learning 
Fig. 5. The relationship between synaptic activity and the behaviour of an adaptive individual. (a) Synaptic activity recorded during the trajectory of the robot. Each line corresponds to the instantaneous strength of a synapse measured every $100 \mathrm{~ms}$ Ticks on the timescale represent the measurements. (b) Trajectory of the robot. Points A, B and C correspond to attraction by the black stripe, the switching on of the light and the arrival to the fitness area, respectively.

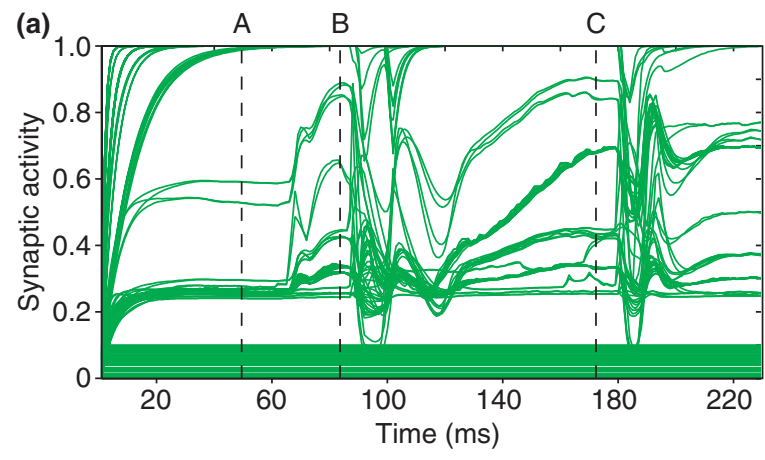

(b)

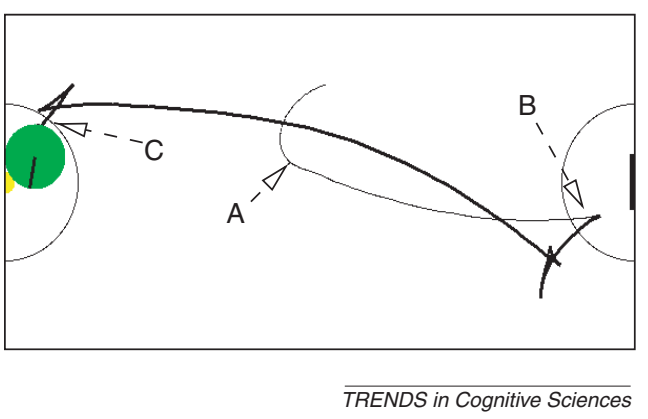

rate. The synaptic strengths were not encoded in the genotype, as in the case of the experiments previously described, but were always initialized to small random values at the beginning of the lifetime of the individual. As soon as the robot begins to move and sensory signals flow through the network, synaptic strengths can change according to the genetically encoded properties and activations of the presynaptic and post-synaptic neurons. The precise balance between weighted signals required to drive the motor neurons in a coordinated fashion had to be learned during lifetime according to genetically specified learning rules.

The analysis of the evolved individuals and the comparison with a control experiment in which the synaptic strengths were directly encoded in the genotype and individuals were not allowed to adapt during lifetime, showed that plastic outperformed non-plastic individuals. Moreover, plastic individuals showed a superior ability to adapt to varied environmental conditions or to a different body structure (achieved by transferring the evolved individual into a larger and geometrically different robot) than non-plastic individuals.

The analysis of the synaptic activity of the evolved controllers while the robot moved in the environment showed that several synapses kept changing during the behavioural sequence (Fig. 5). This pattern of change was characterized by three major events in which most synapses transited into new states. These events are labelled as A, B and C in Fig. 4, and correspond to three clearly different behavioural stages. Event A marks the point when most changing synapses reach a temporarily stable state from their initial random values and the robot displays a smooth trajectory towards the black stripe corresponding to the light switch. Event B is in-between the end of a minor transition and the beginning of a major transition, and corresponds to the point when the robot switches the light on. Finally, event $\mathrm{C}$ marks the end of another period of relatively long change and the beginning of another major temporary transition, and corresponds to the moment when the robot enters the fitness area for the first time (because the robot does not receive information from the floor sensor, it 'knows' that it is on the fitness area by using the pattern of light detected by its sensors). These data suggest that synaptic change corresponds to the acquisition of and switching between different sub-behaviours.

This series of tests indicates that evolved adaptive controllers can develop and/or modify online new behaviours according to the actual environmental conditions. These behaviours are not learned in the classic meaning of the term because they are not necessarily retained forever. For example, the ability to visually locate the black stripe on the wall and move towards it is acquired at a certain stage and disappears once it is no longer necessary. Instead, the attraction towards the light develops early on, even before experiencing the light bulb switched on (notice that light sensors are always activated to some extent by normal ambient light), and is also retained after having reached the fitness area. The graph of synaptic activity shows that after each event there is a major temporary change in all synapses. This always corresponds to the situations when the robot is close to the walls and redevelops online the ability to move away from them.

\section{Conclusion}

An organism is an embodied system that lives and acts in an environment. Unfortunately, in cognitive science this obvious statement is often overlooked even by biologically inspired approaches, such as connectionism, in which cognition is considered as a structure that develops in an abstract environment. It is implicitly assumed that we will know the intrinsic nature of cognition when the laws that govern the dynamics of our brain have been discovered. However, an organism is not only a collection of neurons; it is also immersed in a physical environment and, using its own sensory-motor apparatus, actively selects information from the environment. If we want to understand intelligent life and cognition, it is important to investigate how organisms autonomously interact with their environments; that is, how they behave.

The tendency of underestimating the role of behaviour can be partially explained by considering the difficulty of analysing and designing behavioural systems. Behaviour emerges from the interaction 
between the control system, the body and the external environment, and cannot be analytically described by trying to identify components with rather independent functions. A promising direction describes the control system, the body and the environment as a coupled dynamical system in which each element affects the others [13], and in which the dynamics produced by an element in isolation tend to be significantly different from the dynamics produced by the whole system.

Artificial evolution is an ideal framework for the synthesis and study of systems whose behaviours emerge from the interaction between the control system, body and the environment. In these experiments, as in the case of natural evolution, the control system and the body structure co-evolve in interaction with the environment and selective reproduction is based on the emerging behaviour.

Another research topic that can be successfully tackled with this methodology is the study of lifetime adaptation from an evolutionary perspective. As we illustrated in the previous section, artificial evolution generates individuals that exploit the interaction between the phylogenetic and ontogenetic processes occurring at different timescales. Also in this case, one observes that once the two processes are left free to interact between themselves and with other elements (such as the environment) they deeply affect each other in order to improve the adaptive behaviour of the evolving individuals.

The analysis of the solutions that emerge from these experiments may help us to understand how the interactions between such different entities are exploited in natural organisms, and how to develop artifacts with the robustness, adaptability and flexibility of natural organisms.
The contribution of this body of research to the understanding of natural systems is two-fold. First, experiments conducted by evolving robots in specific environmental conditions can help us to understand how natural organisms solve similar problems in similar environmental conditions. Clearly the results obtained by analysing evolved robots do not necessarily have a direct relevance for explaining how natural organisms produce similar behaviour. However, they can be used to develop new models of adaptive behaviour and cognition that, through experimental analysis, may prove better than existing explanations.

Alternatively, evolutionary robotics experiments may allow us to understand general principles that regulate natural systems. Examples include an improved understanding of the role of sensory-motor coordination [19,21,24]; of the importance of preadaptation and of the ability to adapt 'on the fly' to the current environmental conditions [37,38,42,43]; and of the advantages that arise from the interaction between evolution and lifetime adaptation [25,35].

Evolutionary robotics also has several drawbacks. From an engineering perspective, the most serious problem is the amount of time needed to conduct an evolutionary process on physical robots. From a scientific perspective, the most important challenge is the identification of methods of encoding information into the genotype that are suitable to produce incremental and open-ended evolutionary processes. The extent to which this research will impact our understanding of natural organisms and our ability to develop artifacts with the robustness, adaptability and flexibility of natural organisms will ultimately depend on the extent to which we will be able to tackle these challenges.

\section{References}

1 Cliff, D. et al. (1993) Explorations in evolutionary robotics. Adapt. Behav. 2, 73-110

2 Nolfi, S. and Floreano, D. (2000) Evolutionary Robotics: The Biology, Intelligence, and Technology of Self-Organizing Machines, MIT Press

3 Holland, J.H. (1975) Adaptation in Natural and Artificial Systems, University of Michigan Press

4 Schwefel, H.P. (1995) Evolution and Optimum Seeking, John Wiley \& Sons

5 Koza, J.R. (1992) Genetic Programming: On the Programming of Computers by Means of Natural Selection, MIT Press

6 Brooks, R.A. (1991) Intelligence without reason. In Proc. 12th Int. Joint Conf. Artif. Intell. (Mylopoulos, J. and Reiter, R., eds), Morgan Kaufmann

7 Varela, F.J. et al. (1991) The Embodied Mind: Cognitive Science and Human Experience, MIT Press

8 Hendriks-Jansen, H. (1996) Catching Ourselves in the Act, MIT Press

9 Clark, A. (1997) Being There: Putting Brain, Body and World Together Again, MIT Press

10 Pfeifer, R. and Scheier, C. (1999) Understanding Intelligence, MIT Press

11 Franceschini, N. (1997) Combined optical, neuroanatomical, electrophysiological and behavioral studies on signal processing in the fly compound eye. In Biocybernetics of Vision: Integrative Mechanisms and Cognitive Processes (Taddei-Ferretti, C., ed.), World Scientific

12 Ashby, W.R. (1952) Design for a Brain, Chapman and Hall

13 Beer, R. (1995) A dynamical systems perspective on agent-environment interaction. Artif. Intell. $72,173-215$

14 Dill, M. et al. (1993) Visual pattern recognition in Drosophila involves retinotopic matching. Nature 365, 751-753

15 Thelen, E. and Smith, L.B. (1994) A Dynamics Systems Approach to the Development of Cognition and Action, MIT Press

16 Chiel, H.J. and Beer, R.D. (1997) The brain has a body: adaptive behavior emerges from interactions of nervous system, body and environment. Trends Neurosci. 20, 553-557

17 Braitenberg, V. (1984) Vehicles, MIT Press

18 Bajcsy, R. (1988) Active perception. Proc. IEEE 76, 996-1005

19 Scheier, C. and Pfeifer, R. (1995) Classification as sensorimotor coordination: a case study on autonomous agents. In Advances in Artificial Life (Proc. 3rd Eur. Conf. Artif. Life) (Moran, F. et al., eds), Springer-Verlag
20 Nolfi, S. (1996) Adaptation as a more powerful tool than decomposition and integration. In Proc. Workshop Evol. Comput. Machine Learn. (13th Int. Conf. Machine Learn.) (Fogarty, T. and Venturini, G., eds), University of Bari, Italy

21 Nolfi, S. Power and limits of reactive agents. Neurocomputing (in press)

22 Mondada, R. et al. (1993) Mobile robot miniaturization: a tool for investigation in control algorithms. In Proc. 3rd Int. Symp. Exper. Robots (Yoshikawa, T.Y. and Miyazaki F., eds), Springer-Verlag

23 Nolfi, S. and Parisi, D. (1993) Self-selection of input stimuli for improving performance. In Neural Networks and Robotics (Bekey, G.A., ed.), Kluwer Academic Publishers

24 Scheier, C. et al. (1998) Embedded neural networks: exploiting constraints. Neural Netw. 11, 1551-1596

25 Nolfi, S. and Parisi, D. (1997) Learning to adapt to changing environments in evolving neural networks. Adapt. Behav. 5, 99-105

26 Slocum, A.C. et al. (2000) Further experiments in the evolution of minimally cognitive behavior: from perceiving affordances to selective attention. In From Animals to Animats 6 (Proc. 6th Int. Conf. Simulation Adapt. Behav.) (Meyer, J.A. et al., eds), MIT Press 
27 Michelsen, A. et al. (1994) Physics of directional hearing in the cricket gryllus bimaculatus. J. Comp. Physiol. Ser. A 175, 153-162

28 Webb, B. (1996) A robot cricket. Sci. Am. 275, 94-99

29 Pfeifer, R. (2000) On the relation among morphology and materials in adaptive behavior. In From Animals to Animats 6: Proc. 6th Int. Conf. Simulation Adapt. Behav. (Meyer, J.A. et al., eds), MIT Press

30 Harvey, I. et al. (1994) Seeing the light: artificial evolution, real vision. In From Animals to Animats 3: Proc. 3rd Int. Conf. Simulation Adapt. Behav. (Cliff, D. et al., eds), MIT Press

31 Lichtensteiger, L. and Eggenberger, P. (1999) Evolving the morphology of a compound eye on a robot. In Proc. 3rd Eur. Workshop Adv. Mobile Robots (Eurobot '99), IEEE Press
32 Lund, H.H. et al. (1997) Evolving robot morphology. In Proc. 4th Int. Conf. Evol. Comput., IEEE Press

33 Funes, P. and Pollack, J. (1998) Evolutionary body building: adaptive physical designs for robots. Artif. Life 4, 337-357

34 Lipson, H. and Pollack, J.B. (2000) Automatic design and manufacture of robotic lifeforms. Nature 406, 974-978

35 Nolfi, S. and Floreano, D. (1999) Learning and evolution. Auton. Robots 7, 89-113

36 Todd, P.M. and Miller, G.F. (1991) Exploring adaptive agency II: simulating the evolution of associative learning. In From Animals to Animats: Proc. 1st Int. Conf. Simulation Adapt. Behav. (Meyer, J.A. and Wilson, S.W., eds), MIT Press

37 Floreano, D. and Nolfi, S. (1997) Adaptive behavior in competing co-evolving species.
In Proc. 4th Eur. Conf. Artif. Life. (Husband, P. and Harvey, I., eds), MIT Press

38 Urzelai, J. and Floreano, D. Evolution of adaptive synapses: robots with fast adaptive behavior in new environments. Evol. Comput. (in press)

39 Hinton, G.E. and Nowlan, S.J. (1987) How learning guides evolution. Complex Syst. 1, 495-502

40 Nolfi, S. et al. (1994) Learning and evolution in neural networks. Adapt. Behav. 3, 5-28

41 Nolfi, S. (1999) How learning and evolution interact: the case of a learning task which differs from the evolutionary task. Adapt. Behav. 7, 231-236

42 Husbands, P. et al. (1999) Better living through chemistry: evolving GasNets for robot control. Connection Sci. (10) 3-4, 185-210

43 Nolfi, S. and Floreano, D. (1998) Co-evolving predator and prey robots: do 'arm races' arise in artificial evolution? Artif. Life 4, 311-335

\title{
Structure and function of auditory cortex: music and speech
}

\author{
Robert J. Zatorre, Pascal Belin and Virginia B. Penhune
}

\begin{abstract}
We examine the evidence that speech and musical sounds exploit different acoustic cues: speech is highly dependent on rapidly changing broadband sounds, whereas tonal patterns tend to be slower, although small and precise changes in frequency are important. We argue that the auditory cortices in the two hemispheres are relatively specialized, such that temporal resolution is better in left auditory cortical areas and spectral resolution is better in right auditory cortical areas. We propose that cortical asymmetries might have developed as a general solution to the need to optimize processing of the acoustic environment in both temporal and frequency domains.
\end{abstract}

Music and speech represent the most cognitively complex uses of sound by the human species. These two domains share a number of properties, including the fact that they take advantage of modulations of acoustic parameters specifically for informationbearing purposes. Moreover, both music and speech are characterized by their generative nature: that is, complexity is built up by rule-based permutations of a limited number of discrete elements (phonemes or tones) to yield meaningful structures (words or melodies), which in turn are subject to further hierarchical organization resulting in more complex entities (such as sentences or songs) [1]. Apart from these formal considerations, music and speech show other interesting similarities. For example, both

Robert J. Zatorre*

Pascal Belin

Virginia B. Penhune Montreal Neurological Institute, 3801 University St Montreal, Quebec, Canada H3A 2B4.

*e-mail:

robert.zatorre@mcgill.ca show specific and relatively fixed developmental time courses [2], and all known human societies make use of both speech and music, regardless of technological sophistication [3].

The foregoing ideas do not imply that music and speech necessarily share either a similar underlying cognitive or neural representation (for further discussion see Refs [4,5]), but they do suggest that both might derive from certain functional properties of our auditory nervous system. Put another way, given that all normal humans seem to be capable of relatively sophisticated musical and speech functions in the absence of explicit training, then it follows that these cognitive-behavioral skills are likely to be related to the functional organization of the human auditory nervous system. Just as a bat's ability to use echolocation is related to the unique organization of its auditory system, so the human nervous system may be considered as being organized such that it enables people to readily understand speech and music of the culture in which they are raised. This article explores what this organization might be, with the aim of throwing light upon the neural mechanisms responsible for the low-level perceptual input stage that is relevant for music and speech, and, conversely, taking advantage of speech and music to understand the function of the auditory cortex.

Speech versus music: different acoustic features? Before considering the evidence regarding neural specializations, it is useful to consider some relevant acoustic properties of speech and music. Perhaps the most obvious differences between the two relate to the fact that speech is produced by a single 'instrument' - the human voice, whereas music can be produced by practically anything capable of generating sound, including, of course, the voice. However, certain acoustic parameters have been 